\title{
PER Analysis for Cooperative Multi-Hop Transmission Protocol over Nakagami- $m$ Fading Channels
}

\author{
Tran Trung Duy $\cdot$ Hyung Yun Kong
}

\begin{abstract}
In this paper, we propose a novel protocol called a Multi-hop Diversity Transmission protocol in which the retransmission is realized by a relay that is Nearest to a current Source (MDTNS). We derive the mathematical expressions of the packet error rate (PER) and the average number of transmissions over Nakagami-m fading channels, and verify them by Monte Carlo simulations. The simulation results show that the MDTNS protocol improves the performance of the network in terms of PER when compared to the Multi-hop Diversity Transmission protocol in which the retransmission is done by a relay that is Nearest to Destination (MDTND) and to the conventional multi-hop transmission (CMT) protocol.
\end{abstract}

Key words: Packet Error Rate, Multi-Hop Transmission, Nakagami- $m$ Distribution, Automatic Repeat Request (ARQ).

\section{I . Introduction}

Cooperative wireless communication [1] [5] has gained much attention as an efficient method for mitigating the effects of a fading channel. However, most of the work related to cooperative communication has been developed on single-hop networks. Recently, multihop transmission protocols using cooperative diversity [6] [8] have been proposed and analyzed. In these protocols, the terminals on the primary route are allowed to receive the signals from previous terminals. However, the implementation of these cooperative multi-hop transmission protocols is complex and not very feasible in practice. First, relays have to restore all versions of the received data, which can require huge storage capacity. Second, although these protocols can significantly enhance performance compared with the conventional multi-hop transmission protocol, the end-to-end delay and the power consumption, which are important criteria in multi-hop transmission, cannot be reduced.

The ARQ technique [9] is a method in which the retransmission is employed whenever the destination receives an erroneous packet. Therefore, the reliability is enhanced but at a cost of more end-to-end delay. In addition, in a block fading environment [10], the delay can be transformed into a diversity gain, which is a more important benefit.

In this paper, we propose a multihop technique that we have named MDTNS, which is embedded with automatic repeat request-based (ARQ) diversity transmission to enhance the reliability of data transmission and to reduce delay time. We assume that a route between the source and the destination is established by the network layer and that each relay lying on this route can receive the packet from all previous nodes. In the proposed protocol, a packet is discarded as soon as it is received incorrectly at each node; thus, the combining techniques such as Maximal Ratio Combining or Selection Combining are not employed at the receiver. In addition, as mentioned above, when the destination fails in receipt of the packet, the retransmitted packet can be originated from a relay that is nearest to the current transmitting node and that has successfully decoded.

The transmission will terminate whenever the destination can receive the packet without errors. Therefore, the proposed protocol can reduce the end-to-end delay due to some omitted relays, while it significantly improves performance compared with the CMT protocol by employing diversity transmission. Unlike the MDTNS protocol, the MDTND protocol, which is also proposed in this paper, selects the relay nearest to the destination to retransmit the packet to the destination. Although the MD-

Manuscript received November 2, 2011 ; Revised March 14, 2012 ; Accepted May 3, 2012. (ID No. 20111102-032J)

Department of Electronic Engineering, University of Ulsan, Ulsan, Korea.

Corresponding Author : Hyung-Yun Kong (e-mail : hkong@mail.ulsan.ac.kr)

This is an Open-Access article distributed under the terms of the Creative Commons Attribution Non-Commercial License (http://creativecommons.org/licenses/ by-nc/3.0) which permits unrestricted non-commercial use, distribution, and reproduction in any medium, provided the original work is properly cited. 
TND protocol further reduces the transmission time, it achieves a lower diversity gain than does the MDTNS protocol. As a result, the MDTNS protocol outperforms the MDTND protocol at high Signal-to-Noise Ratio values. The performance of these protocols is evaluated by theoretical results and simulation results in Nakagami- $m$ fading channels.

The rest of the paper is organized as follows. The system model and the proposed scheme are described in Section II. In Section III, the performance analysis of the MDTNS protocol is discussed. In Section IV, we show the simulation results and Section $V$ concludes the paper.

\section{System Model}

In Fig. 1, we present an established route that consists of nodes $N_{1}, N_{2}, N_{3}, \cdots, N_{M}, N_{M+1}$, where $N_{1}$ is the source, $N_{M+1}$ is the destination, and relays $N_{i}(2 \leq i \leq M)$ are numbered according to their distance to the destination, with relay $N_{2}$ being furthest and relay $N_{M}$ being the nearest.

Consider that the channels between two nodes are subjected to block and flat Nakagami- $m$ fading, in which the channel remains unchanged during a packet period, but is allowed to change independently from packet to packet. The Nakagami- $m$ distribution function proposed by Nakagami [11] can model a variety of fading scenarios, including the Rayleigh distribution and one-sided Gaussian distribution.

Each node is assumed to have a single half duplex radio and a single antenna. The symbol received at a receiver $\mathrm{R}$ due to the transmission of a transmitter $\mathrm{T}$ for packet $\mathrm{s}$ is given by

$$
y_{T R}=\sqrt{P} h_{T R} s+\eta_{R}
$$

where $P$ is the average transmitted power of the transmitter $\mathrm{T}, \mathrm{s}$ is transmitted packet, $\eta_{R}$ is AWGN noise with variance $N_{0}, h_{T R}$ captures the block, and flat Nakagami- $m$ fading is a channel between nodes $\mathrm{T}$ and $\mathrm{R}$.

Therefore, the instantaneous signal-to-noise ratio (SNR) of the packet is expressed by

$$
\gamma_{T R}=P\left|h_{T R}\right|^{2} / N_{0}
$$

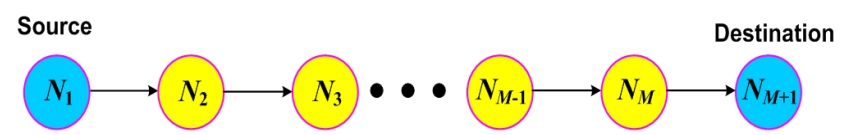

Fig. 1. An $M$-hop route from the source $N_{1}$ to the destination $N_{M+1}$.
Because $h_{T R}$ has the Nakagami- $m$ distribution, $\gamma_{T R}$ has the following probability density function (PDF):

$$
f_{\gamma_{T R}}\left(\gamma_{T R}, m_{T R}\right)=\frac{m_{T R}^{m_{T R}}}{\Gamma\left(m_{T R}\right) \gamma_{T R}^{-m_{T R}}} \gamma_{T R}^{m_{T R}-1} \exp \left(-m_{T R} \frac{\gamma_{T R}}{\overline{\gamma_{T R}}}\right)
$$

where $m_{T R} \geq 1 / 2$ is a parameter describing the fading severity of channel between transmitter $\mathrm{T}$ and receiver $\mathrm{R}, \overline{\gamma_{T R}}$ is the expected value of $\gamma_{T R}$, and $\Gamma($.$) is the$ Gamma function.

In this paper, for simplicity of analysis, $m_{T R}$ is assumed to receive an integer value and $m_{T R}$ is unchanged for all channels; i.e., $m_{T R}=m$.

We account for path loss by modeling the expected value of the channel coefficient between nodes $T$ and $R$ as a function of distance between two nodes [12], as

$$
\overline{\gamma_{T R}}=\frac{P}{N_{0}} d_{T R}^{-\beta}=S N R d_{T R}^{-\beta}
$$

where $\beta$ is called the path loss exponent and varies from 2 to 6 on the basis of channel environment, and $d_{T R}$ is the distance between nodes $\mathrm{T}$ and $\mathrm{R}$.

\section{2-1 The CMT Protocol}

Initially, the source node $N_{1}$ transmits a packet attached with cyclic redundancy check (CRC) bits. Assume that the CRC-based error detection is perfect. The use of the CRC technique imparts two advantages: the first is that the CRC codes are sufficiently reliable and the second is that the overhead created by CRC is negligible, compared with the number of payload bits. The packet is next transmitted sequentially from node $N_{i}$ to node $N_{i+1}(2 \leq i \leq M)$. If node $N_{i+1}$ cannot decode the packet correctly, it requests a retransmission from the node $N_{i}$. We should note that, in order to avoid a large number of retransmissions, the retransmission time of each node should be limited by a threshold value $k$. This means that the packet will be dropped at the $i$-th hop if the $k$-th retransmission from node $N_{i}$ fails.

\section{2-2 The MDTND Protocol}

Initially, the source broadcasts the packet to the destination and all relays. The destination and all the relays then decode the received packet. If the destination decodes correctly, it sends an acknowledge message (ACK) to inform the source and all the relays to start a new transmission. Otherwise, the destination generates 
a negative-acknowledge message (NACK) to request a retransmission. The relay that is nearest to the destination and that decodes the packet successfully will then become a new transmitter for the destination. Unlike the conventional ARQ protocols, in which the source repeats the transmission, the retransmission in this protocol is originated by the relays. The benefits of retransmission from the relay are: first, that the relay is nearer to the destination than the source; hence, the packet is relayed to the destination with a higher probability of success. Second, the relay channel is dependent on the source-destination channel; thus, the retransmission will increase the diversity gain. Assume that the retransmission is started from node $N_{i}$ which successfully decodes the packet received from the source and is nearest to the destination. The destination and all relays between node $N_{i}$ and the destination can then receive and decode the packet. If the destination does not decode successfully again, it requests a retransmission from relay $N_{j}(i<j<M+1)$, which is nearest to the destination and receives the packet successfully. In the case where there is no relay $N_{j}$ that successfully decodes the packet, node $N_{i}$ will retransmit this packet. Similarly to the CMT protocol, we limit the maximum number of retransmissions for each node $N_{i}$ by the threshold value $k$.

In order to realize this protocol, the channel access is assumed to be Time Division Multiple Access (TDMA), where the nodes cannot simultaneously receive and transmit in the same channel. In addition, after receiving the NACK message from the destination, the relay nearest to the destination (i.e., relay $N_{M}$ ) will send a control signal to inform the decoding status. If node $N_{M}$ decodes correctly, it will forward the packet to the destination and the remaining relays will keep silent. Otherwise, node $N_{M-1}$, in turns, sends the control signal and it will become the new source if it decodes correctly. The above channel access procedure is continued until a relay is selected for the retransmission or the transmitting node retransmits the packet, provided that the remaining retransmission times of this node are larger than zero.

\section{2-3 The MDTNS Protocol}

Unlike the MDTND protocol, the destination in the MDTNS will request the relay that is nearest to the transmitting node $N_{i}(2 \leq i \leq M)$ and that decodes the packet successfully, in order to retransmit a packet erroneously received by the destination. Similarly to the MDTND protocol, after receiving the NACK message from the destination, the nodes $N_{j}, j=\{i+1, i+2, \ldots, M\}$ will sequentially send the control signal to inform the decoding status and this procedure is started from node $N_{i+1}$. In the case where no relay $N_{j}$ decodes successfully, either node $N_{i}$ will retransmit the packet or the packet will be dropped. In addition, with the MDTNS protocol, when a relay receives the packet without errors, it will not receive any further packets and will wait until it, in turn, transmits this packet to the destination.

\section{Performance Analysis}

We first must introduce some notations used in this section. Let $\overline{P E R}_{i j}^{\mathrm{DT}}$ denote the average PER of the direct transmission from node $i$ to node $j, \overline{P E R}_{i j}^{k \text {-CMT }}$, $\overline{P E R}_{i j}^{k \text {-MDTND }}$, and $\overline{P E R}_{i j}^{k \text {-MDTNS }}$ as the average PER of a $(j-i)$-hop route that uses the CMT protocol, the MDTND protocol, and the MDTNS protocol, respectively, to relay a packet from source node $i$ to destination node $j$, provided that each node in these routes is allowed to retransmit $k$ times.

With the instantaneously received SNR $\gamma_{i j}$, according to (2), we have the exact PER when the Forward Error Correction (FEC) technique is not used, as

$$
P E R_{i j}^{\mathrm{DT}}=1-\left(1-\operatorname{BER}\left(\gamma_{i j}\right)\right)^{L}
$$

where $B E R\left(\gamma_{i j}\right)$ is the bit error rate when the received SNR is $\gamma_{i j}$ and $L$ is the length of the packet.

Because this exact expression is very complicated for analysis, we use the approximation proposed in [9]:

$$
\operatorname{PER}_{i j}^{\mathrm{DT}}\left(\gamma_{i j}\right) \approx \begin{cases}1 & \text { if } 0<\gamma_{i j}<\gamma_{p} \\ \alpha \exp \left(-g \gamma_{i j}\right) & \text { if } \gamma_{i j} \geq \gamma_{p}\end{cases}
$$

where $\left(\alpha, g, \gamma_{p}\right)$ are found in [9] and the threshold $\gamma_{p}$ is set such that $\alpha \exp \left(-g \gamma_{p}\right)=1$.

Applying the above approximated expression, the average PER in the Nakagami- $m$ fading channels can be calculated by

$$
\begin{aligned}
\overline{P E R}_{i j}^{\mathrm{DT}} & =\int_{0}^{\infty} P E R_{\gamma_{i j}}(\gamma) f_{\gamma_{i j}}(\gamma) d \gamma_{i j} \\
& =\frac{\gamma\left(m, m \frac{\gamma_{p}}{\bar{\gamma}}\right)}{\Gamma(m)}+\left(\frac{m}{m+g \bar{\gamma}}\right)^{m} \frac{\alpha \Gamma\left(m,\left(\frac{m}{\bar{\gamma}}+g\right) \gamma_{p}\right)}{\Gamma(m)}
\end{aligned}
$$

where $\gamma(x, y)=\int_{0}^{y} e^{-t} t^{x-1} d t$ is the lower incomplete gamma function and $\Gamma(x, y)=\int_{y}^{\infty} e^{-t} t^{x-1} d t$ is the upper incomplete gamma function. 


\section{3-1 The CMT Protocol}

In this protocol, the average PER of hop $i$ between nodes $N_{i}$ and $N_{i+1}$ is calculated as

$$
\overline{\operatorname{PER}}_{i, i+1}^{k-\mathrm{CMT}}=\left(\overline{\operatorname{PER}}_{i, i+1}^{\mathrm{DT}}\right)^{k+1}
$$

Next, we consider the $M$-hop route, while all of the hops are independent. Thus, the end-to-end PER of this route can be given as

$$
\overline{P E R}_{1, M+1}^{k-\mathrm{CMT}}=1-\prod_{i=1}^{M}\left(1-\overline{\operatorname{PER}}_{i, i+1}^{k-\mathrm{CMT}}\right)
$$

\section{3-2 The MDTND Protocol}

The average PER where the source transmits the packet incorrectly to the destination can be calculated recursively as follows:

$$
\begin{aligned}
& \overline{P E R}_{1, M+1}^{k-\mathrm{MDTND}}= \\
& =\overline{P E R}_{1, M+1}^{\mathrm{DT}}\left(\prod_{t=2}^{M} \overline{P E R}_{1, t}^{\mathrm{DT}} P_{1-(k-1)}^{\mathrm{MDTND}}+\sum_{t=2}^{M} Q_{t} \overline{P E R}_{t, M+1}^{k-\mathrm{MDTND}}\right)
\end{aligned}
$$

where,

$$
Q_{t}= \begin{cases}\left(1-\overline{P E R}_{1, t}^{\mathrm{DT}}\right) \prod_{h=t+1}^{M} \overline{P E R}_{1, h}^{\mathrm{DT}} & \text { if } t \leq M-1 \\ \left(1-\overline{P E R}_{1, M}^{\mathrm{DT}}\right) & \text { if } t=M\end{cases}
$$

and

$$
P_{1-(k-1)}^{\mathrm{MDTND}}=\left\{\begin{array}{l}
\overline{P E R}_{1, M+1}^{\mathrm{DT}}\left(\begin{array}{l}
\prod_{t=2}^{M} \overline{P E R}_{1, t}^{\mathrm{DT}} P_{1-(k-2)}^{\mathrm{MDTND}} \\
\left.+\sum_{t=2}^{M} Q_{t} \overline{P E R}_{t, M+1}^{k-\mathrm{MDTND}}\right)
\end{array}\right) \text { if } k \geq 1 \\
1 \quad \text { if } k=0(12)
\end{array}\right.
$$

In (10), if all relays between the source $N_{1}$ and the destination $N_{M+1}$ cannot successfully receive the packet from the source $N_{1}$, the source retransmits the packet with the number of retransmission decreased by 1 . Hence, $P_{1-(k-1)}^{\mathrm{MDTND}}$ represents the average PER of the first retransmission of the source and $P_{1-(k-1)}^{\mathrm{MDTND}}$ is calculated recursively, as in (12).

\section{3-3 The MDTNS Protocol}

Similar to the MDTND protocol, the end-to-end average PER for the MDTNS protocol is also calculated recursively by

$$
\begin{aligned}
& \overline{P E R}_{1, M+1}^{k \text {-MDTNS }}= \\
& =\overline{P E R}_{1, M+1}^{\mathrm{DT}}\left(\prod_{t=2}^{M} \overline{P E R}_{1, t}^{\mathrm{DT}} P_{1-(k-1)}^{\mathrm{MDTNS}}+\sum_{D} Q_{D} \overline{P E R}_{D}^{k \text {-MDTNS }}\right)
\end{aligned}
$$

where $P_{1-(k-1)}^{\mathrm{MDTNS}}$ is calculated by

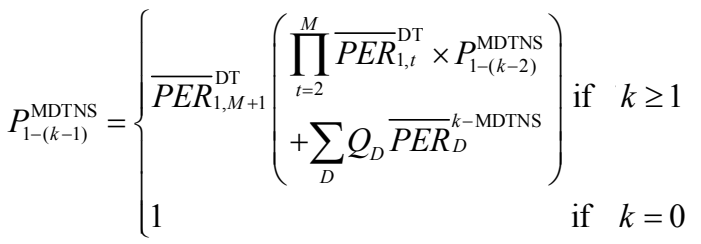

In (13), (14), $D$ is defined as the set of relays correctly decoding the packet received from the source $N_{1}$. It is noted that the size of $D$, say $|D|$, varies from 1 to $M$. Assume that $D=\left\{N_{k_{1}}, N_{k_{2}}, \ldots, N_{k_{n}}\right\}$ with $k_{n}>k_{n-1}$ $>\ldots>k_{1}$, the probability $Q_{D}$ is calculated by

$$
Q_{D}=\prod_{N_{j} \notin D}\left(\overline{P E R}_{N_{1}, N_{j}}^{\mathrm{DT}}\right) \prod_{N_{j} \in D}\left(1-\overline{P E R}_{N_{1}, N_{j}}^{\mathrm{DT}}\right)
$$

In this protocol, node $N_{k_{1}}$ will be selected to retransmit the packet to the destination $N_{M+1}$. Then, the set of nodes that are allowed to decode the packet from node $N_{k_{1}}$ is $D_{k_{1}}=\left\{N_{j} \mid N_{j} \notin D, k_{1}<j<M+1\right\}$. In order to calculate the probability $\overline{P E R}_{D}^{k \text {-MDTNs }}$, we consider the three following cases.

For the first case, the size of $D_{k_{1}}$ is equal to zero, and we have

$$
\overline{\operatorname{PER}}_{D}^{k \text { MDTNS }}= \begin{cases}\overline{P E R}_{k_{1}, M+1}^{\mathrm{DT}} \overline{\operatorname{PER}}_{D_{1}}^{k \text {-MDTNS }} & \text { if }|D|>1 \\ \left(\overline{P E R}_{k_{1}, M+1}^{\mathrm{DT}}\right)^{k+1} & \text { if }|D|=1\end{cases}
$$

For the second case, in the set $D_{k_{1}}$ contains at least one node, but set $D$ has only one member, i.e., $D=$ $\left\{N_{k_{1}}\right\}$. In this case, $\overline{P E R}_{D}^{k \text {-MDTNS }}$ is calculated as

$$
\begin{aligned}
\overline{P E R}_{D=\left\{N_{k_{1}}\right\}}^{k-\mathrm{MDTNS}}=\overline{\operatorname{PER}}_{k_{k_{1}, M+1}^{\mathrm{DT}} \times} & \left(\prod_{N_{j} \in D_{L_{1}}} \overline{P E R}_{k_{k_{1}}, j}^{\mathrm{DT}} P_{k_{1}-(k-1)}^{\mathrm{MDTNS}}+\sum_{D_{1}} Q_{D_{1}} \overline{P E R}_{D_{1}}^{k-\mathrm{MDTNS}}\right)
\end{aligned}
$$

where $P_{k_{1}-(k-1)}^{\mathrm{MDTN}}$ is calculated similarly to $P_{1-(k-1)}^{\mathrm{MDTNS}}, D_{1}$ represents a set of relays that belong to the set $D_{k_{1}}$ and successfully decode the received packet from node $N_{k_{1}}$. In addition, $Q_{D_{1}}$, and $\overline{P E R}_{D_{1}}^{k \text { MDTNS }}$ are calculated similarly to $Q_{D}$ in (15) and $\overline{P E R}_{D}^{k \text {-MDTNS }}$ in (16), respectively. 
For the third case, at least one relay belongs to the set $D_{k_{1}}$ while the set $D$ has more than one member, i.e., $|D| \geq 2$. In this case, $\overline{P E R}_{D, N_{M+1}}^{k-\text { IDTNS }}$ is given as follows

$$
\begin{aligned}
& \overline{P E R}_{D}^{k-\mathrm{MDTNS}}=\overline{P E R}_{k_{1}, M+1}^{\mathrm{DT}} \\
& \quad \times\left(\prod_{N_{j} \in D_{1}} \overline{P E R}_{k_{1}, j}^{\mathrm{DT}} \overline{P E R}_{D \backslash\left\{N_{k_{1}}\right\}}^{k-\mathrm{MDTNS}}+\sum_{D_{1}} Q_{D_{1}} \overline{P E R}_{D_{2}}^{k-\mathrm{MDTNS}}\right)
\end{aligned}
$$

Unlike the second case, when all the nodes in set $D_{k_{1}}$ cannot decode correctly, then the next relay in the set $D$, i.e., $N_{k_{2}}$, will retransmit the packet to the destination. Otherwise, let us denote $D_{1}$ as set of the relays that belong to the set $D_{k_{1}}$ and that successfully decode the received packet from node $N_{k_{1}}$. Now, the set of the nodes that successfully decode the received packet either from the source $N_{1}$ or from relay $N_{k_{1}}$ is determined as $D_{2}=D \cup D_{1} \backslash\left\{N_{k_{1}}\right\}$. Also, $D_{1}$ and $D_{2}$ are random sets and the probabilities $Q_{D_{1}}$ and $\overline{P E R}_{D_{2}}^{k \text {-MDTNS }}$ are determined similarly to the above cases.

\section{Simulation Results}

In this section, we provide some numerical results of the outage probabilities that have been developed in Section III and verify them with Monte Carlo simulations.

In this section, we use the Monte Carlo simulation to verify theoretical results. The packet size is 1,080 bits/ packet, and the corresponding fixed parameters $\alpha$, g, and $\gamma_{p}$ are $67.7328,0.9819$, and $6.3281 \mathrm{~dB}$ [9]. We assume that the transmit power $P$ is the same for every

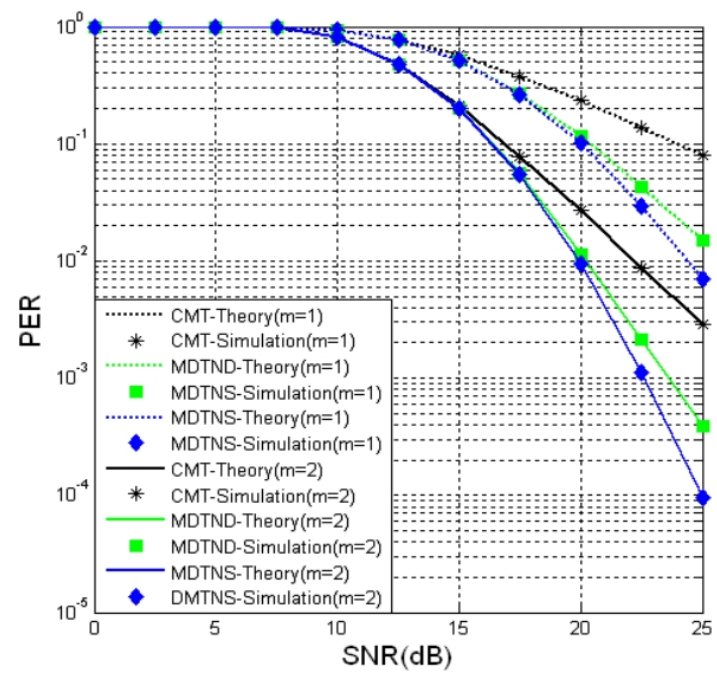

Fig. 2. The end-to-end PER as a function of the SNR with different values of $m$.

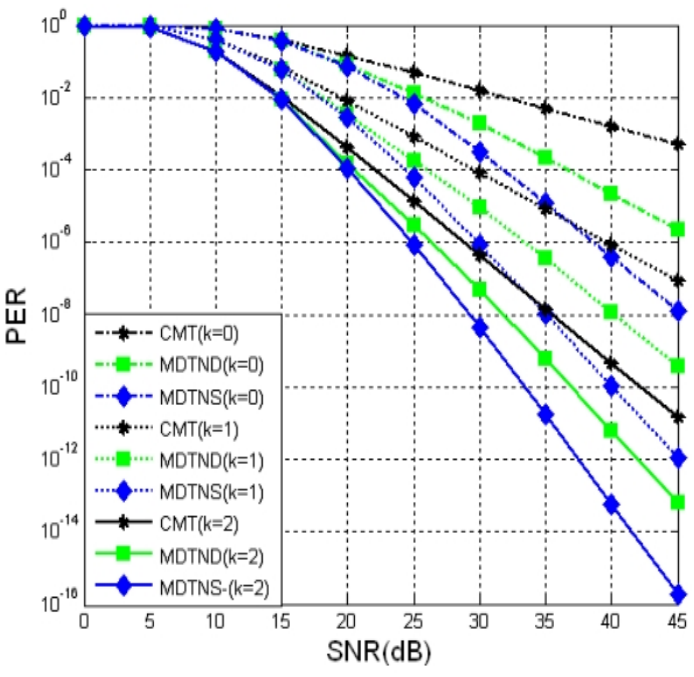

Fig. 3. The end-to-end PER as a function of SNR with the different number of retransmissions.

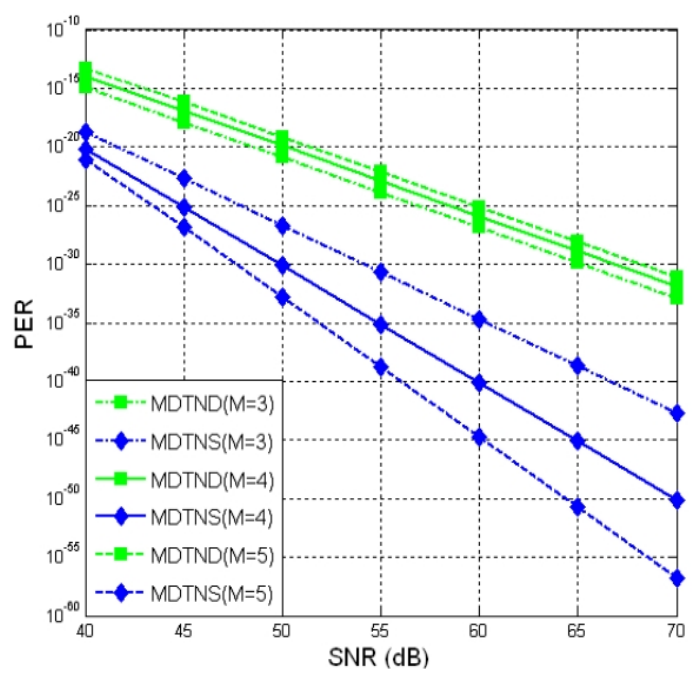

Fig. 4. The end-to-end PER as a function of SNR with the different number of hops.

transmitting node, including the source and all relays. Furthermore, we also consider the line network where all of the nodes are on the same line and the distance between two adjacent nodes is equal to 1 and the path loss coefficient equals 4 for all simulations.

Fig. 2 shows that the theoretical results and simulation results of the end-to-end PER match very well for all cases. This simulation is implemented on the 5-hop networks without retransmission $(k=0)$ and on different fading channels; i.e., Rayleigh fading $(m=1)$ and $\mathrm{Na}-$ kagami-2. At high SNR, the MDTNS protocol obtains the best performance compared to the remaining protocols, while the CMT protocol shows the worst performance. In addition, the performance of all protocols is better if the value of $\mathrm{m}$ is increased. 
Fig. 3 shows the theoretical effect of the number of allowed retransmission times $k$ on the system performance. In this figure, the value of $m$ is fixed by 1 (Rayleigh fading channel) and the number of hops $M$ is set to 3 . The figure shows that when the number of retransmissions $k$ is increased, all protocols gain higher diversity order. Among these, the MDTNS protocol achieves the highest diversity gain, while the MDTND protocol always has one degree higher than the diversity order of the CMT protocol in the Rayleigh fading channel $(m=1)$. This is due to the fact that the MDTNS protocol has more relays taking part in the cooperation. However, the result is that the MDTNS protocol uses more power than does the MDTNS protocol.

In Fig. 4, we present the end-to-end PER of the MDTND protocol and the MDTNS protocol at very high SNR values in order to determine the diversity order of these two protocols. In this figure, we set the number of retransmissions $k$ to 1 , the value of $m$ to 2, and the number of hops $M$ varies from 3 to 5 . The MDTND protocol has a diversity order of 6 for all values of $M$, while the MDTNS protocol has diversity order of 8,10 , and 12 , corresponding to the number of hops of 3,4 , and 5 .

\section{Conclusion}

In this paper, we propose a novel protocol called a multi-hop diversity transmission protocol where the retransmission is realized by a relay that is nearest to the current source (MDTNS). In the proposed protocol, the relay node that is nearest to the source and that decodes the source packet correctly will retransmit a packet erroneously received by the destination. In order to evaluate the performance of the proposed protocol, we derived the mathematical expressions to calculate the end-to-end PER and the average number of transmissions and then we verified the accuracy of the derivation by simulating. The simulation results show that the MDTNS protocol improves the performance of the system, when compared to the MDTND and CMT protocols at high SNR region. Furthermore, our proposal can reduce power consumption and shorten the delay from end-to-end due to some bypassed relays.

This work was supported by University of Ulsan, School of Excellence in Electrical Engineering.

\section{References}

[1] J. N. Laneman, D. N. C. Tse, and G. W. Wornell,
"Cooperative diversity in wireless networks: Efficient protocols and outage behavior," IEEE Transactions on Information Theory, vol. 50, no. 12, pp. 3062-3080, Dec. 2004.

[2] T. E. Hunter, A. Nosratinia, "Diversity through coded cooperation," IEEE Transactions on Wireless Communications, vol. 5, no. 2, pp. 283-289, Feb. 2006.

[3] A. Bletsas, A Khisti, D. P. Reed, and A Lippman, "A simple cooperative diversity method based on network path selection," IEEE Journal on Selected Areas in Communications, vol. 24, no. 3, pp. 659-672, Mar. 2006.

[4] B. An, T. T. Duy, and H. -Y. Kong, "A cooperative transmission strategy using entropy-based relay selection in mobile ad-hoc wireless sensor networks with Rayleigh fading environments," KSII Transactions on Internet and Information Systems, vol. 3, no. 2, pp. 147-162, Apr. 2009.

[5] T. T. Duy, H. -Y. Kong, "Outage analysis of cooperative transmission in two-dimensional random networks over Rayleigh fading channels," Journal of the Korean Institute of Electromagnetic Engineering and Science, vol. 11, no. 4, pp. 262-268, Dec. 2011.

[6] J. Boyer, David D. Falconer, and H. Yanikomeroglu, "Multihop diversity in wireless relaying channels," IEEE Transactions on Communications, vol. 52, no. 10, pp. 1820-1830, Oct. 2004.

[7] Golnaz Farhadi, Norman C. Beaulieu, "Selective decode-and-forward relaying scheme for multi-hop diversity transmission systems," GLOBECOM 2007, pp. 4385-4390.

[8] J. Zhang, T. M. Lok, "Performance comparison of conventional and cooperative multihop transmission," IEEE Wireless Communications and Networking Conference, Apr. 2006.

[9] Q. Liu, S. Zhou, and G. B. Giannakis, "Cross-layer combining of adaptive modulation and coding with truncated ARQ over wireless links," IEEE Transactions on Wireless Communications, vol. 3, pp. 17461755, 2004.

[10] R. Knopp, P. A. Humblet, "On coding for block fading channels," IEEE Transactions on Information Theory, vol. 46, no. 1, pp. 189-205, Jan. 2000.

[11] M. Nakagami, "The m-distribution-A general formula for intensity distribution of rapid fading," Statistical Methods in Radio Wave Propagation, W.G. Hoffman, Ed., Oxford, U.K, Pergamon, 1960.

[12] T. T. Duy, B. An, and H. -Y. Kong, "A novel cooperative-aided transmission in multi-hop wireless networks," IEICE Transactions on Communications, vol. E93.B, no. 3, pp. 716-720, Mar. 2010. 


\section{Tran Trung Duy}

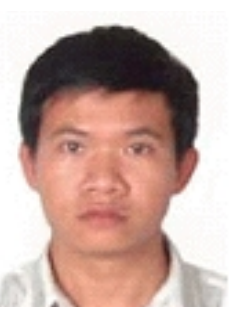

received Telecommunications Engineering from Ho Chi Minh City University of Te the B.E. degree in Electronics and technology, Vietnam, in 2007. He is currently working toward the Master degree in the Department of Electrical Engineering, University of Ulsan, Korea. His major research interests are mobile ad-hoc networks, wireless sensor networks, cooperative communications, cooperative routing, cognitive radio, combining techniques.

\section{Hyung Yun Kong}

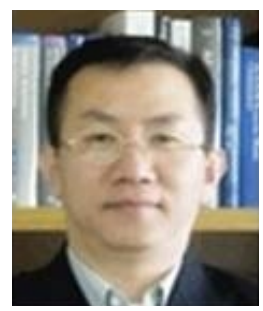

received the M.E. and Ph.D. degrees in electrical engineering from Polytechnic University, Brooklyn, New York, USA, in 1991 and 1996, respectively, He received a $\mathrm{BE}$ in electrical engineering from New York Institute of Technology, New York, in 1989. Since 1996, he has been with LG electronics Co., Ltd., in the multimedia research lab developing PCS mobile phone systems, and from 1997 the LG chairman's office planning future satellite communication systems. Currently he is a Professor in electrical engineering at the University of Ulsan, Korea. His research area includes channel coding, detection and estimation, cooperative communications, cognitive radio and sensor networks. He is a member of IEEK, KICS, KIPS, IEEE, and IEICE. 